\title{
Cosmological constant and quintessence
}

\section{Z. Stuchlík}

\author{
Department of Physics, Faculty of Philosophy and Science, Silesian University at Opava \\ Bezručovo nám. 13, CZ-74601 Opava, Czech Republic \\ E-mail: zdenek.stuchlik@fpf.slu.cz, URL:http://uf.fpf.slu.cz/
}

\begin{abstract}
The role of an effective repulsive cosmological constant (a false vacuum or quintessence) in the very early inflationary expansion and the recent accelerated stage of our universe is reviewed, and it is shown that the relict cosmological constant can significantly influence accretion phenomena in recent universe.

Je podán přehled role efektivní kosmologické konstanty (falešného vakua nebo kvintesence) ve velmi rané inflační expanzi a současné urychlené expanzi našeho vesmíru. Je ukázáno, že reliktní kosmologická konstanta může silně ovlivňovat akreční procesy v současné epoše evoluce vesmíru.
\end{abstract}

\section{Introduction}

Recent cosmological observations suggest that immediately after the big bang our universe experienced an extremely accelerated inflationary expansion, while at the present era the universe entered a second stage of accelerated expansion [Wang et al., 2000]. The rate of present acceleration is many orders of magnitude smaller than during the inflation in the very early universe, i.e., we can call it a microinflationary expansion.

The inflationary cosmology is the first theory within which it is possible to predict the structure of the universe on large scale, based on causal physics, representing thus a promising way for connecting fundamental physics with experiment. It enables a natural explanation for almost all of the problems of the standard cosmology [Linde, 1990]. Especially, the mechanism by which initial quantum vacuum fluctuations develop into the large scale structure of the universe is tested by recent observations in deep detail [Bahcall et al., 1999].

Usually, the inflationary models assume that during inflation potential energy density of a scalar (inflaton) field $\phi$ dominates the energy-momentum tensor, $T_{\mu \nu} \sim g_{\mu \nu} V(\phi)$, i.e., we must have slowly rolling inflaton field with $\frac{1}{2} \dot{\phi}^{2} \ll V(\phi)$. The limiting case $\dot{\phi}=0$ corresponds to de Sitter space with a repulsive cosmological constant.

The current accelerated expansion can be explained either by a positive vacuum energy $V$ (cosmological constant), or by a slowly rolling, quintessential scalar field. The acceleration mechanism is the same for the inflaton and quintessential fields. The inflaton field decays giving rise to the standard decelerated expansion. The behavior of the quintessential field in future is the key to the fate of our universe.

\section{Inflationary cosmology}

The inflationary cosmology is based on the hypothesis that there was an extremely short time interval, just after the Big Bang, beginning at some time $t_{\mathrm{i}}$ and ending at the 'reheating time' $t_{\mathrm{R}}$ during which the scale 
factor $R(t)$ grows exponentially. This period is called inflationary or 'de Sitter,' because the expansion is driven by energy of a false vacuum, equivalent to a repulsive cosmological constant.

The inflationary cosmology represents an appropriate combination of General Relativity and Quantum Field Theory and is based on the behavior of the inflaton scalar field $\phi$ in the expanding universe. The energy density and pressure of such a field are given by $(\cdot$ denotes $\mathrm{d} / \mathrm{d} t)$ :

$$
\begin{aligned}
& \rho=\frac{1}{2} \dot{\phi}^{2}+\frac{(\nabla \phi)^{2}}{2 R^{2}(t)}+V(\phi), \\
& p=\frac{1}{2} \dot{\phi}^{2}-\frac{(\nabla \phi)^{2}}{6 R^{2}(z)}+V(\phi) .
\end{aligned}
$$

Its time evolution in a nearly homogeneous region is determined by an equation resembling the one describing motion with friction of a ball on a slightly declined desk

$$
\ddot{\phi}+3 H \dot{\phi}=\frac{\mathrm{d} V(\phi)}{\mathrm{d} \phi}
$$

where $H \equiv \dot{R} / R$ is the Hubble parameter and $3 H \dot{\phi}$ is the frictional term. The evolution of the universe is determined by the equations for acceleration term

$$
\frac{\ddot{R}}{R}=-\frac{4 \pi}{3}(\rho+3 p)
$$

and the energy density evolution

$$
\dot{\rho}=-3 H(\rho+p) \text {. }
$$

Now, it is clear that in a homogeneous region of dimension $\ell_{\mathrm{i}}$, where $\nabla \phi \sim 0$, the inflationary exponential expansion can occur, if $\phi^{2} / 2 \ll V(\phi)$. Then the nearly vacuum equation of state $p \sim-\rho$ is satisfied, and the nearly de Sitter expansion will occur with acceleration given by

$$
\frac{\ddot{R}}{R} \sim \frac{8 \pi}{3} \rho \sim \frac{8 \pi}{3} V(\phi),
$$

and with nearly constant energy density $\dot{\rho} \sim 0$. (The accelerated expansion will occur for an equation of state with $p<-\rho / 3$.) The expansion factor grows exponentially as

$$
R(t) \sim \exp (H(\phi) t)
$$

where

$$
H(\phi) \approx\left[\frac{8 \pi}{3} V(\phi)\right]^{1 / 2} \sim\left(\frac{\Lambda}{3}\right)^{1 / 2} \sim \text { const. }
$$

It follows from the evolution equation (3) that the scalar field will roll slowly $(\ddot{\phi} \ll 3 H \dot{\phi}, 3 H \dot{\phi} \sim \mathrm{d} V / \mathrm{d} \phi)$ for a long time compared to the characteristic time $\Delta t_{\mathrm{H}} \sim H^{-1}$ yielding thus the inflationary expansion of the initially homogeneous region. The inflation is finished at the moment of reheating occurring when $\ddot{\phi} \gtrsim 3 H \dot{\phi}$. The scalar field then oscillates around its minimum corresponding to the true vacuum state and releases its energy to ordinary matter via interaction terms in the Lagrangian [Linde, 1990]. The minimal inflation, necessary to explain the problems of the standard cosmology, is given by the inflationary blow up of the order

$$
\ell_{\mathrm{f}} \sim \ell_{\mathrm{i}} \exp \left(\frac{t_{\mathrm{i}}-t_{\mathrm{R}}}{\delta t_{H}}\right) \sim \ell_{\mathrm{i}} \exp (100) .
$$

The inflationary cosmology then explains, e.g., the problem of the prime mover, because the inflaton field and its repulsive gravitational effect are the reason of the expansion of the universe, and predicts the velocity of the expansion to be corresponding to the flat universe, since the parameter $\Omega=\rho / \rho_{\text {crit }} \approx 1\left(\rho_{\text {crit }}=3 H / 8 \pi G\right)$ due to the evolution law

$$
\Omega-1=\frac{k}{H^{2} \exp (2 H(\phi))} \sim 0,
$$

independently of the parameter $k=-1,0,1$ determining character of the space geometry of the universe. 
During inflation, a causal (particle) horizon is present with dimension of the order of $\ell_{\mathrm{c}} \sim H^{-1}(\phi)$. Simultaneously, there is also an event cosmological horizon, analogous to the event horizon of a black hole, present in the inflationary universe. Its dimension $\sim H^{-1}(\phi)$. With the existence of the event horizon a temperature $T_{\mathrm{c}} \sim H / 2 \pi$ is connected. Under the event horizon, the classical fluctuations of the inflaton field are exponentially damped because no-hair cosmological theorem holds [see Eqs (1) and (2)]. On the other hand, the quantum (vacuum) fluctuations of the field are related to the event horizon by $\delta \phi / \phi \sim H(\phi) / 2 \pi$, being continuously created and stretched exponentially during inflationary era, giving rise to density perturbations of matter latter, in the standard period of the evolution of the universe. Once their physical wavelength equals the Hubble radius, the vacuum oscillations freeze out, the quantum state is squeezed. The amplitude of these perturbations, given by $H(\phi)$, remains frozen when the wavelength exceeds the causal horizon, and starts a new evolution after crossing back the causal horizon during the standard decelerated expansion of the universe. Since $H(\phi)$ is approximately constant during the period of inflation, this mechanism of quantum fluctuations freezing out and becoming classical density fluctuations of the order of [Linde, 1990]

$$
\frac{\delta \rho}{\rho} \sim \frac{H^{2}}{\dot{\phi}},
$$

leads to nearly scale invariant spectrum of fluctuations, as suggested by Zel'dovich to explain the large scale structure of the universe. These predictions of the inflationary cosmology are confirmed with growing evidence by measurements of the character of the anisotropies of the Cosmic Microwave Background [Bahcall et al., 1999].

The first inflationary models were related to the GUT theories of elementary particles, with the inflaton field being the Higgs field responsible for breaking of the symmetry between the strong and electroweak interactions. In these models the inflation begins from the symmetry state of $\phi \sim 0$ at $t_{\mathrm{i}} \sim 10^{-34} \mathrm{~s}$ with $t_{\mathrm{R}} \sim 10^{-32} \mathrm{~s}$, so they just fit the condition for the minimal inflation. However, they failed in explaining magnitude of primordial density fluctuations of matter, estimated to be $\delta \rho / \rho \sim 10^{-5}$.

Therefore, models of primordial inflation based on the supergravity theory were developed by the CERN group. The main advantage of these models is that they are able to solve the problem of density fluctuation magnitude. For primordial inflation the inflaton field starts its evolution from the symmetry state with $\phi \sim 0$ at $t_{\mathrm{i}} \sim 10^{-42} \mathrm{~s}$, comparable to the Planck time when effect of quantum gravity are important, and the inflationary blow up exceeds for many orders the minimal value of $\exp (100)$.

At present, there is a lot of variants of the inflationary models. The chaotic inflation is a generic model, because it assumes the inflaton field so weakly coupled to the matter that it does not start at thermal equilibrium $(\phi \sim 0)$. Most of the phase space of the initial conditions of values of $\phi$ takes values much higher than the Planck scale. Therefore, the inflationary blow up takes extremely high values, e.g., $\exp \left(10^{6}\right)$. Moreover, the quantum fluctuations can have magnitude higher than the change of the field given by its classical evolution, leading to the idea of an eternal, self-regenerating universe containing even parts with $\phi$ permanently growing due to the quantum fluctuations. According to this scenario, the universe is highly inhomogeneous, containing parts with physical properties and even dimensionality very different in comparison with those of the part we are living in. The inflationary cosmology can then naturally be related to the concept of spontaneous creation of the universe from nothing [Linde, 1990].

The most recent development of the inflationary cosmology is connected to the $\mathrm{M} /$ string theory and the brane approach to the universe according to which our spacetime is a 4-dimensional brane in a higher-dimensional spacetime. (However, it is worth to mention some non-inflationary string alternatives to cosmology: the Pre-bigbang model of Veneziano [Veneziano, 1991], and the idea of the Ekpyrotic universe [Khoury et al., 2001] based on colliding branes.) 


\section{$3 \quad$ Tracker fields}

A wide range of independent observations strongly indicate that vast majority, about two thirds, of the energy in the recent state of the universe is in the form of 'dark energy' having the extraordinary property of the false vacuum of the inflationary cosmology: its gravity effects are repulsive, not attractive. This fact is directly confirmed by recent observations of high-redshift supernovae.

The dark energy can be in the form of vacuum energy with equation of state $p=-\rho$. Nevertheless, there is unexplained the problem of miraculous tuning of the vacuum energy to the extremely small, but nonzero value at early stages of expansion of the universe, when matter was by 100 orders of magnitude denser than the vacuum energy.

However, there is another possibility that the dark energy is basically similar to the energy of false vacuum of the inflationary cosmology. We call this dark energy 'quintessence' or 'fifth element' and it can be described in the present era, by the equation of state $p=w \rho, w \in(-1,-1 / 3)$; usually we assume $w \gtrsim-1$.

A natural way to obtain a quintessential scalar field is connected to brane solution of the M/string theory. The presence of the curled up extra dimensions or nearby branes (three-dimensional surfaces to which the matter and known physical fields are connected) act like a field having the quintessential behavior if the radius of curl up or the gap between branes changes slowly.

The quintessential field could solve the problem of fine tuning in the framework of so called tracker fields proposed in 1999 [Armendariz-Picon et al., 2000]. The equations describing tracker fields have classical attractor behavior similar to those known from some chaotic systems. Evolution of tracker fields converges to the same result for a wide range of initial conditions. It locks into a track on which its energy density remains a nearly constant fraction of the density of radiation (and matter). The tracker fields imitate radiation during the early stages of the universe, although it is completely different in its nature. The tracking occurs because the radiation and matter density determine the cosmic expansion rate, which controls the rate at which the quintessence energy density changes.

The energy density of the quintessential field tracks the radiation and matter density for most of the history of the universe. At beginning, it represents a small fraction of the critical density, but the fraction grows slowly and, ultimately, it overtakes the matter density. Of course, an important question remains - namely, why the acceleration phase of the expansion of the universe begins at the particular moment when the galaxies were formed. One answer can be proposed in the spirit of the anthropic principle. Another answer is that the accelerated stage is triggered by a natural event in the history of the universe. Such a natural event is given by the shift of the radiation dominated to the matter dominated, decelerated expansion of the universe. A special model of the ' $\mathrm{k}$-essence' or kinetic-energy-driven quintessence [Armendariz-Picon et al., 2000] triggers the behaviour of the quintessential field from the tracker mode in which it follows the energy density of radiation to the false vacuum mode in which it evolves to the gravitationally repulsive state with dominant potential term and negligible kinetic energy term. The sudden and sharp fall of the kinetic energy of the quintessential field is driven by the onset of matter dominance.

The potential of the quintessential field must give rise to both the tracker mode and the false vacuum mode. In order to obtain the tracker (false vacuum) mode, an exponential (polynomial) character is necessary. Generally, the potential can be presented in the form

$$
V(\phi)=\left[(\phi-B)^{\gamma}+A\right] \exp (-\lambda \phi) .
$$

The parameters $A, B, \gamma, \lambda$ must be chosen appropriately to enable explanation of all the successes of the standard and inflationary cosmology, e.g., the primordial nucleosynthesis, or the character of primordial fluctuations reflected at the anisotropies of cosmic relic radiation. Recent observations will give very restrictive limitations of the values of the parameters. However, an ultimate goal is to obtain such a quintessential field from first principles, i.e., from the $\mathrm{M} /$ string theory. 
The ultimate fate of the universe will be given by the behavior of the quintessential field, not by the geometry. If the false vacuum state of the quintessential field will appear to be stable, the accelerated expansion will continue and the cosmos will be increasingly hostile, leading to isolated islands of matter. If the false vacuum state will be unstable, the quintessence could decay through nucleation of bubbles which could collide in a firework leading to a new, highly structured, inhomogeneous universe.

\section{Astrophysical implications of the relict false vacuum state}

The role of a quintessential false vacuum state or a vacuum energy in astrophysical processes can be estimated by studies of the character of black-hole solutions with a nonzero repulsive cosmological constant $\Lambda>0$. The role of $\Lambda>0$ can be quite relevant in properties of test particle motion, or the character of equilibrium configurations of perfect fluid orbiting a black hole. The results have clear impact on properties of accretion disks around giant black holes in active galactic nuclei and quasars [Stuchlík, 2002]. These properties can be appropriatelly illustrated by using the optical geometry [Hledík, 2002]. The other strong influence of $\Lambda>0$ could be expected in the framework of Rees-Sciama effect on anisotropies of cosmic radiation background [Stuchlík, 2002].

In the following we shall shortly discuss the most interesting effect of positive $\Lambda$ on the structure of thick accretion disks [Stuchlík et al., 2000]. The rotating fluid with 4-velocity $U^{\mu}=\left(U^{t}, 0,0, U^{\phi}\right) ; U^{t}=U^{t}(r, \theta), U^{\phi}=U^{\phi}(r, \theta)$ is characterized by the angular velocity and the specific angular momentum density $\Omega=U^{\phi} / U^{t}, \ell=-U_{\phi} / U_{t}$, and by energy density $\epsilon$ and pressure $p$. The Euler equation implies the Boyer condition determining surfaces of constant pressure by equipotential surfaces of the potential $W(r, \theta)$ :

$$
\int_{0}^{p} \frac{\mathrm{d} p}{p+\epsilon}=W_{\text {in }}-W ; \quad W_{\text {in }}-W=\ln \left(U_{t}\right)_{\text {in }}-\ln \left(U_{t}\right)+\int_{\ell_{\text {in }}}^{\ell} \frac{\Omega \mathrm{d} \ell}{1-\Omega \ell} .
$$

The relevant physical properties are given by the simplest case of marginally stable configurations of $\ell(r, \theta)=$ const, when

$$
W(r, \theta)=\ln U_{t}(r, \theta) .
$$

The equipotential surfaces $\theta=\theta(r)$ are then given by the formula

$$
\frac{\mathrm{d} \theta}{\mathrm{d} r}=-\frac{\partial U_{t} / \partial r}{\partial U_{t} / \partial \theta}
$$

For the Schwarzschild-de Sitter spacetimes, it takes the form

$$
\frac{\mathrm{d} \theta}{\mathrm{d} r}=\tan \theta \frac{\left[r\left(1-y r^{3}\right) \sin ^{2} \theta-\left(1-\frac{2}{r}-y r^{2}\right)^{2} \ell^{2}\right] r}{\left(r-2-y r^{3}\right)^{2} \ell^{2}},
$$

where the cosmological parameter $y \equiv \Lambda M^{2} / 3$. In the astrophysically relevant case of $y<12 / 15^{4}$, we arrive to the following conclusions (see Fig. 1):

1. Equipotential surfaces with an outer cusp exist enabling outflow of matter and angular momentum from the accretion disk by violation of mechanical equilibrium. Such an outflow is impossible for accretion disk around an isolated black hole with $\Lambda=0$. Moreover, there is a critical equipotential surface with both the inner and outer cusp - in such a case the mechanical violation of disk equilibrium caused accretion inflow and outflow with the same efficiency.

2. There is possibility of strong collimation effect on jets escaping along symmetry axis of the disk after crossing so called static radius, i.e., in regions where influence of the cosmological repulsion dominates attraction of the black hole. 


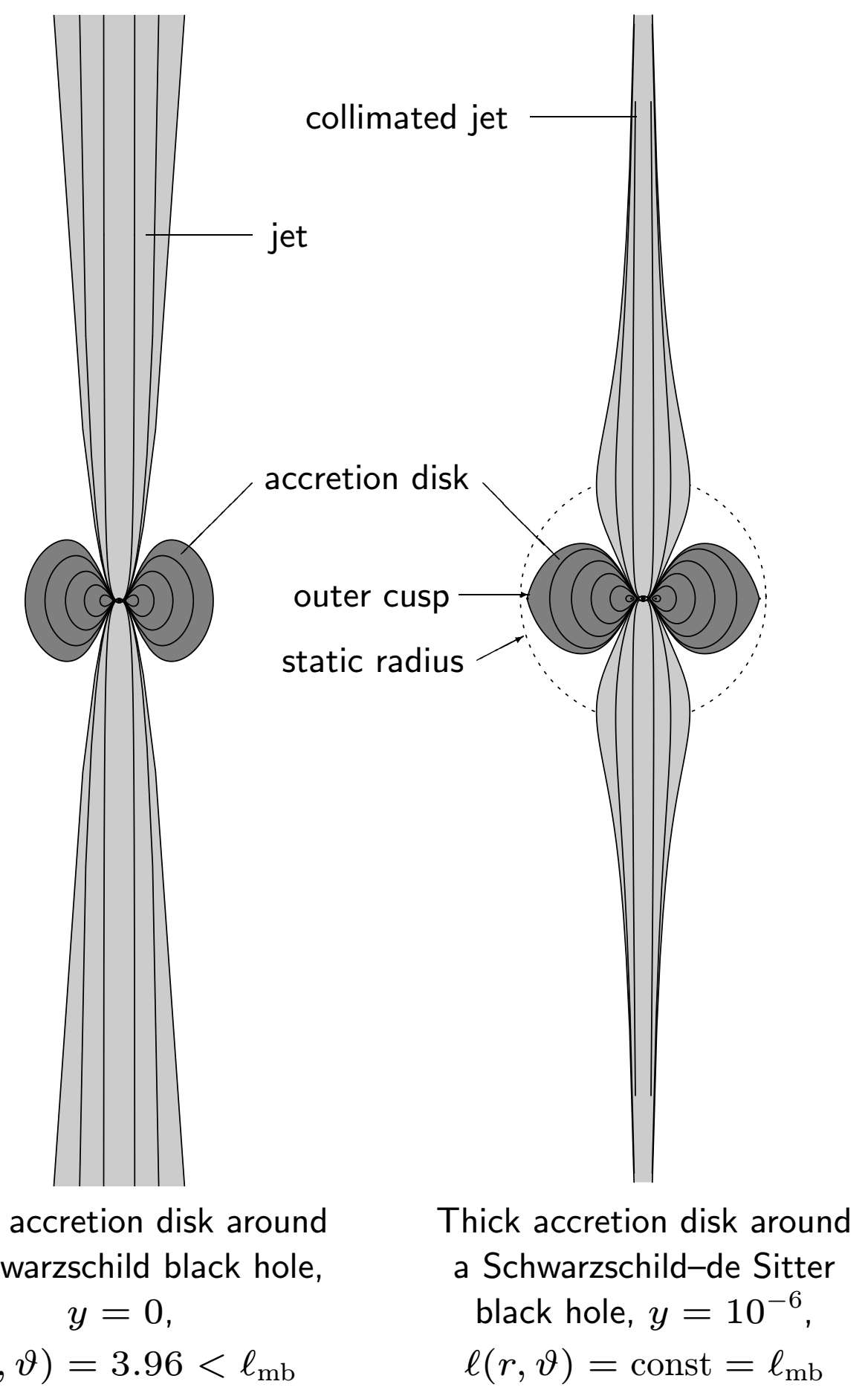

Figure 1: The structure of an accretion disk with a jet is compared in meridian sections. The radial coordinate is expressed in units of $M$ but the logarithmic scale is not used here since we are interested in the regions near the static radius where both the outer cusp and the collimation effect are evident. Taken from Ref. [Stuchlík et al., 2000]. 


\section{Conclusions}

Numerical studies of the influence of $\Lambda>0$ on accretion processes in the disk regime indicate quite interesting coincidence. Extend of accretion disks around black holes of $M \sim 10^{9} M_{\odot}$ is about $50 \mathrm{kpc}$ which is comparable with maximum extension of large galaxies. Moreover, the collimation effect of $\Lambda>0$ could be relevant in these situations because the largest observed jets reach distances $\sim 200 \mathrm{kpc}$, clearly exceeding dimensions of the 'seed' galaxy [Stuchlík, 2002].

We expect that planned observations will help to better understanding of the nature of quintessence and its behaviour in future, determining the fate of our universe.

\section{Acknowledgements}

The present work was supported by the GAČR grant No. 202/02/0735 and by the Committee for collaboration of the Czech Republic and CERN. The author would like to express his gratitude to the Theory Division of CERN for perfect hospitality.

\section{References}

[Armendariz-Picon et al., 2000] Armendariz-Picon, C., Mukhanov, V., and Steinhardt, P. J. (2000). Dynamical solution to the problem of a small cosmological constant and late-time cosmic acceleration. Phys. Rev. Lett., 85(21):4438.

[Bahcall et al., 1999] Bahcall, N., Ostriker, J. P., Perlmutter, S., and Steinhardt, P. J. (1999). The cosmic triangle: Revealing the state of the universe. Science, 284:1481-1488.

[Hledík, 2002] Hledík, S. (2002). Optical geometry, inertial forces, and embedding diagrams. In [Semerák et al., 2002], pages 161-192.

[Khoury et al., 2001] Khoury, J., Ovrut, B. A., Steinhardt, P. J., and Turok, N. (2001). Ekpyrotic universe: Colliding branes and the origin of the hot big bang. Phys. Rev. D, 64(12):123522 (24 pages).

[Linde, 1990] Linde, A. D. (1990). Particle Physics and Inflationary Cosmology. Gordon and Breach, New York.

[Semerák et al., 2002] Semerák, O., Podolský, J., and Žofka, M., editors (2002). Gravitation: Following the Prague Inspiration (A Volume in Celebration of the 60th Birthday of Jiři Bičák), New Jersey, London, Singapore, Hong Kong. World Scientific.

[Stuchlík, 2002] Stuchlík, Z. (2002). The role of a repulsive cosmological constant in astrophysical processes. In [Semerák et al., 2002], pages 27-83.

[Stuchlík et al., 2000] Stuchlík, Z., Slaný, P., and Hledík, S. (2000). Equilibrium configurations of perfect fluid orbiting Schwarzschild-de Sitter black holes. Astronomy and Astrophysics, 363(2):425-439.

[Veneziano, 1991] Veneziano, G. (1991). Phys. Lett. B, 265:287.

[Wang et al., 2000] Wang, L., Caldwell, R. R., Ostriker, J. P., and Steinhardt, P. J. (2000). Cosmic concordance and quintessence. Astrophys. J., 530(1):17-35. 